\title{
Measuring saccade curvature: A curve-fitting approach
}

\author{
CASIMIR J. H. LUDWIG and IAIN D. GILCHRIST \\ University of Bristol, Bristol, England
}

\begin{abstract}
Saccade curvature is becoming a popular measure for detecting the presence of competing saccadic motor programs. Several different methods of quantifying saccade curvature have been employed. In the present study, we compared these metrics with each other and with novel measures based on curve fitting. Initial deviation metrics were only moderately associated with the more widely used metric of maximum curvature. The latter was strongly related to a recently developed area-based measure and to the novel methods based on second- and third-order polynomial fits. The curve-fitting methods showed that although most saccades curved in only one direction, there was a population of trajectories with both a maximum and a minimum (i.e., double-curved saccades). We argue that a curvature metric based on a quadratic polynomial fit deals effectively with both types of trajectories and, because it is based on all the samples of a saccade, is less susceptible to sampling noise.
\end{abstract}

That the eye generally does not take the shortest route when moving to fixate a target, but is subject to idiosyncratic distortions in the trajectory, has been recognized for a long time (see, e.g., Yarbus, 1967). Initial attempts to characterize the curvature found in saccade trajectories focused on how the direction of deviation depended on the location of the saccade target and on what type of saccade was made (Smit \& Van Gisbergen, 1990; Viviani, Berthoz, \& Tracey, 1977). More recent studies have demonstrated that the extent and direction of saccadic curvature is modulated by the instruction to attend to a location other than the saccade target (Sheliga, Riggio, Craighero, \& Rizzolatti, 1995; Sheliga, Riggio, \& Rizzolatti, 1994, 1995; Tipper, Howard, \& Paul, 2001). Curvature also occurs when an irrelevant distractor is presented in close proximity to the saccade target (Doyle \& Walker, 2001). In all of these experiments, saccades curved away from the attended (distractor) location. It has been argued that these distractordependent trajectory modulations are a result of competing saccade programs for the target and distractor locations. Together, these studies have resulted in an increasing interest in using (saccade) curvature as a sensitive measure of distractor interference in saccade targeting. However, different studies have used different methods to quantify curvature, hindering quantitative across-study comparisons.

This work was supported by a University of Bristol Postgraduate Research Scholarship awarded to the first author and by a grant from the Wellcome Trust to the second author. We thank Chris Benton, Christof Koerner, Eugene McSorley, Robin Walker, Jonathan Vaughan, and an anonymous reviewer for their helpful comments and suggestions throughout the preparation of this article. Correspondence should be addressed to C. J. H. Ludwig, Department of Experimental Psychology, University of Bristol, 8 Woodland Rd., Bristol BS8 1TN, England (e-mail: c.ludwig@bristol.ac.uk).
The different metrics employed in previous research are illustrated in Figure 1, which shows the trajectories of two saccades observed in one of our experiments. The left panel shows a saccade with a single curve. The right panel shows a double-curved saccade that clarifies the potential problems faced by the different curvature metrics (strictly speaking, both trajectories contain just a single curve, but have either one maximum or one minimum, or both a maximum and minimum; for convenience, we denote these saccades as single-curved or double-curved saccades).

\section{Initial Direction}

The first metric to be used was the angular difference between the initial direction and the overall direction (Findlay \& Harris, 1984; Van Gisbergen, Van Opstal, \& Roebroek, 1987). The angle between the initial direction computed at a fixed point in the saccade (e.g., after $20 \mathrm{msec}$ in Van Gisbergen et al., 1987) and the overall direction determined at the endpoint of the saccade yields a measure of curvature (marked as an arc in Figure 1).

\section{Initial Average}

Sheliga, Riggio, Craighero, and Rizzolatti (1995) and Sheliga, Riggio, and Rizzolatti (1995) employed the initial average deviation measure (illustrated with black squares in Figure 1). This method is conceptually related to the initial direction metric. They calculated the deviation of each sample in the initial $10 \mathrm{msec}$ of the saccade ${ }^{1}$ by simply subtracting the eye position on the dimension orthogonal to the saccade direction (e.g., horizontal for vertical saccades) from the value on that dimension at the start of the saccade (e.g., horizontal starting position). These deviations were then averaged.

Both the initial direction and the initial average metrics are theoretically appealing, in that they measure the influence of a competing saccade program at the start of the 

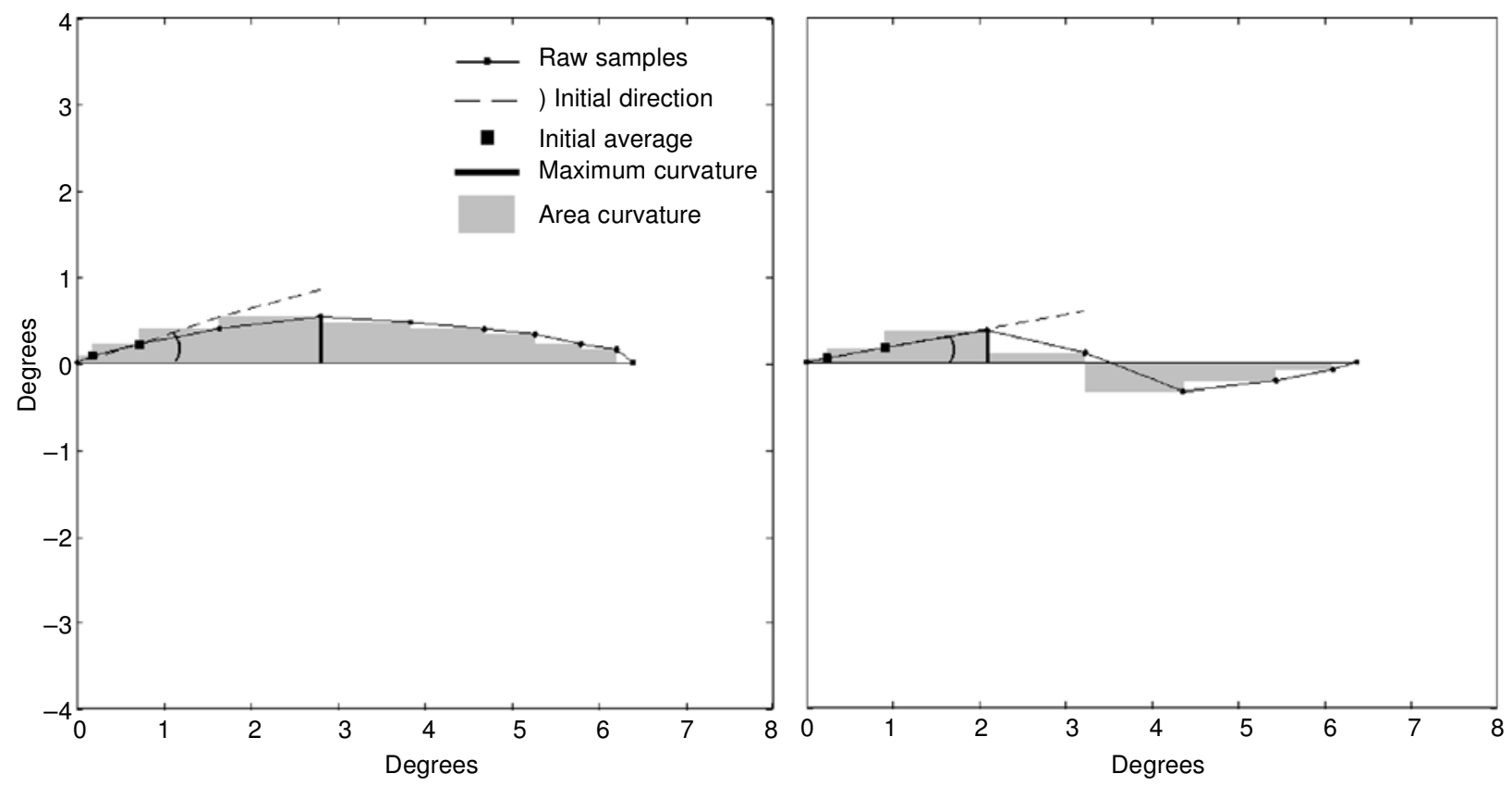

Figure 1. Illustration of the different metrics found in the literature. These metrics are imposed on a single-curved (left) and a doublecurved (right) saccade taken from the data set that was analyzed in this study. The saccades are translated so that the straight line between start and landing positions corresponds to the abscissa. The numbers on the axes represent degrees. See text for details of how the metrics are calculated.

saccade. However, the problem with both methods is that they determine the initial direction after a fixed interval. As a result, saccades with identical trajectories but different velocities of execution will be assigned different amounts of curvature (Smit \& Van Gisbergen, 1990).

\section{Maximum Curvature}

Smit and Van Gisbergen (1990) proposed a maximum curvature measure (illustrated with thick vertical lines in Figure 1), which has been used in the majority of curvature studies since its publication (e.g., Doyle \& Walker, 2001, 2002; McPeek \& Keller, 2001). For each sample, the perpendicular distance from a straight line between the starting point and the endpoint of the saccade is calculated. Signed curvature is defined as the largest absolute perpendicular deviation (e.g., in Figure 1 and in our analyses, a negative value represents a counterclockwise deviation). This metric is less theoretically driven than the measures based on the initial deviation, but it deals effectively with the problem of identical movement trajectories executed at different velocities.

One problem with this method is that it assumes that the point of maximum curvature of a saccade is the best representation of the nature and extent of curvature for that saccade. Although this may not be too serious in the case of a saccade that curves in only one direction (Figure 1, left panel), it is hard to see how a single sample could represent the curvature of a trajectory with both a maximum and a minimum (Figure 1, right panel). In addition, with double-curved saccades, it may be that the point of maximum deviation is located in the second curve of the trajectory. If one assumes (as with the initial deviation metrics) that the influence of a competing eye movement program is to initially drive the eye away from the target, it is the first curve that is of most interest. To the extent to which these double-curved saccades occur in the saccades under study, the maximum curvature method might miss some of the theoretically interesting deviations.

\section{Area Curvature}

To remedy such potential problems, Doyle and Walker (personal communication, September 17, 2001) have recently developed a measure based on the entire trajectory of the eye movement (shaded area of Figure 1). For each sample $n$, the distance covered along the straight path between onset and endpoint since the previous sample $(n-1)$ is multiplied by the perpendicular (signed) deviation of sample $n$. The sum of these segments is taken as the amount of curvature in the saccade. Because the deviations are signed, a double-curved saccade such as that shown in the right panel of Figure 1 would have a curvature close to zero. (With a less symmetrical double-curved saccade, this metric would be biased in the direction of the more pronounced curve.) This method seems to be an effective way of determining the dominant direction and magnitude of the curvature. However, as Figure 1 also demonstrates, by simply multiplying the distance along the abscissa by its associated deviation, each segment alone will either under- or overestimate the area under the curve for the corresponding part of the trajectory. If sac- 
cades are not symmetrically curved, this introduces a systematic bias, because the errors do not cancel each other out.

One potential problem shared by all the curvature metrics discussed above (except the area curvature method) is that the calculations are based on only a subset of the sampled positions in each saccade. This may be particularly problematic when the metric is based on a single point in the trajectory (such as the initial direction and maximum curvature measures). Because eye trackers (especially userfriendly, cost-effective ones) are limited in their spatial and temporal resolution, these metrics can be subject to substantial measurement error. For instance, using the SMI EyeLink system (SensoMotoric Instruments $\mathrm{GmbH}$, Teltow, Germany), we sampled eye position every $4 \mathrm{msec}$ and typically achieved a spatial accuracy of about $0.3^{\circ}$ (the upper limit that we allowed for is $0.5^{\circ}$, after Doyle \& Walker, 2001). In order to achieve a more reliable curvature measure, we have developed a curve-fitting approach. Because all the data in a given saccade contribute to the shape of the curve, these methods are less susceptible to noise.

In this article, we report a systematic comparison of the different curvature metrics. We took a set of eye-movement records from one of our experiments, calculated the different measures, and explored how well they corresponded with each other. Driven by informal observations of doubleand even triple-curved saccades, we further aimed in this study to investigate whether such saccades constituted a distinct subset in the data set. The curve-fitting methods allowed us to test this directly.

\section{METHOD $^{2}$}

\section{Data}

The set of eye movement records was taken from one of our curvature experiments, the results of which will be reported elsewhere. Briefly, the aim of the experiment was to see whether the amount of curvature away from irrelevant distractors was modulated by the target similarity of those distractors (cf. Ludwig \& Gilchrist, 2002). The two possible target locations, $6^{\circ}$ left and right of the central fixation point, were indicated by gray squares ("placeholders"). After $500 \mathrm{msec}$, one of the placeholders turned red and the other turned green. Twelve participants were asked to saccade to the red target. Only the saccades that landed within a $2^{\circ}$ region around the target were subjected to the curvature analysis. On the majority of trials, abrupt-onset distractors appeared either above or below fixation, but only the no-distractor baseline trials were included in the analyses reported here (571 trials pooled over the 12 observers).

\section{Curve Fitting and Monte Carlo Simulation}

Every saccade was translated so that the axis through its starting and landing positions coincided with the abscissa (see Figure 1). Four polynomials were fitted to each saccade: (1) a simple linear function, (2) a quadratic polynomial, (3) a cubic polynomial, and (4) a quartic polynomial. For each polynomial, the $R^{2}$ goodness of fit was calculated for each saccade, and the distribution of these $R^{2}$ values was plotted.

There are two possible explanations for the increase in $R^{2}$ associated with the increase in complexity of the polynomials fitted. The first is that the more complex polynomials genuinely reflect the trajectory of the saccades. For example, an improvement of fit as a result of the introduction of a quadratic component over and above the linear one could mean that saccades are generally not linear. Alternatively, it may be that the increased degrees of freedom simply allow the fitted function to accommodate deviations that are the result of noise. One way to differentiate between these two possibilities is to investigate the spatial distribution of the residuals following curve fitting. If the remaining variability in the trajectory is a result of noise, there should be no systematic relationship between the residuals (observed - predicted deviations) and their locations within the trajectory.

To test the relation between residuals and their locations in the trajectory, we carried out the following simulation. After fitting a polynomial of order $n$ to the raw samples of the saccade, we calculated the residual for each sample. These residuals were then randomly assigned to the predicted deviations of the $n$th polynomial, and the $n+1$ polynomial was fitted (on the reshuffled trajectory). The assumption was that if the remaining variance unaccounted for represents simply noise instead of a systematic component, randomly assigning this noise to different points in the trajectory should not make a difference in the improvement of fit obtained with the higher order $(n+1)$ function. After analyzing the entire data set in this manner, we calculated the median $R^{2}$ values for the quadratic, cubic, and quartic fits. This procedure was run 5,000 times to extract distributions of median $R^{2}$ values. These distributions were used to test directly whether the improvements of $R^{2}$ with increasing degrees of freedom were due to accommodating sampling noise or systematic trajectory components.

\section{Calculation and Comparison of the Metrics}

Again, as a first step, each saccade was translated so that the straight line between start and endpoint coincided with the horizontal axis, and the values on the ordinate indicate the perpendicular deviations from the straight line (as in Figure 1). We then calculated the initial direction ( $8 \mathrm{msec}$ into the saccade, i.e., at the third sample), the initial average (mean of perpendicular deviations at the second and third samples), the maximum curvature, and the area curvature metric for each saccade in the data set. In addition, we derived curvature mathematically from the second- and third-order polynomial fits (quadratic and cubic curvature metrics).

First, the horizontal axis was rescaled so that each saccade started at $x=-1$ and ended at $x=1$. This rescaling leaves the shape of the saccade and the function unaffected, but makes the units of the coefficients of the second-order polynomial meaningful. The quadratic coefficient is a global property of the function and directly indicates the amount of curvature. This amount equals the difference between the predicted deviation at a particular point and the average of the predicted deviations one unit to the left and right of that point (Darlington, 1990). Because of the rescaling of the horizontal axis, curvature is now defined relative to the predicted deviations at the start and endpoint of the saccade. We will show later that these predicted deviations are generally very close to zero. As a result, the quadratic coefficient of the second-order fit approximates the deviation from a straight line between start and endpoint. Furthermore, because this deviation is in pixels, and given the fixed relation between number of pixels and degrees of visual angle, curvature can be expressed in terms of degrees. Thus, the quadratic coefficient can be used as a direct estimate of the amount of curvature in units that are meaningful.

With regard to the third-order fit, the meanings of the coefficients themselves are less immediately obvious, and we adopted a different approach. Again, the fitted function is essentially treated as the saccade itself, and two points of maximum curvature (i.e., the maximum and minimum of the function) can be calculated mathematically. The perpendicular deviation at these points relative to the straight line between the predicted start and endpoint of the saccade was determined. With two points of maximum curvature, one could now simply choose the larger of the two (making this metric similar to the maximum curvature metric, but basing it on the entire trajectory) or focus on either the first or the second deviation, whichever 
Table 1

Means and Ranges of the Correlations Between the Curvature Metrics

\begin{tabular}{|c|c|c|c|c|c|c|}
\hline Metric & $\begin{array}{c}\text { Initial } \\
\text { Direction }\end{array}$ & $\begin{array}{c}\text { Initial } \\
\text { Average }\end{array}$ & $\begin{array}{l}\text { Maximum } \\
\text { Curvature }\end{array}$ & $\begin{array}{c}\text { Area } \\
\text { Curvature }\end{array}$ & $\begin{array}{l}\text { Quadratic } \\
\text { Curvature }\end{array}$ & $\begin{array}{c}\text { Cubic } \\
\text { Curvature }\end{array}$ \\
\hline Initial direction & 1 & $\begin{array}{c}.91 \\
(.82-.96)\end{array}$ & $\begin{array}{c}.73 \\
(.48-.90)\end{array}$ & $\begin{array}{c}.77 \\
(.66-.91)\end{array}$ & $\begin{array}{c}.72 \\
(.56-.84)\end{array}$ & $\begin{array}{c}.72 \\
(.51-.86)\end{array}$ \\
\hline Initial average & & 1 & $\begin{array}{c}.73 \\
(.49-.89)\end{array}$ & $\begin{array}{c}.76 \\
(.60-.89)\end{array}$ & $\begin{array}{c}.70 \\
(.52-.83)\end{array}$ & $\begin{array}{c}.71 \\
(.43-.83)\end{array}$ \\
\hline Maximum curvature & & & 1 & $\begin{array}{c}.94 \\
(.86-.98)\end{array}$ & $\begin{array}{c}.94 \\
(.85-.97)\end{array}$ & $\begin{array}{c}.94 \\
(.84-.98)\end{array}$ \\
\hline Area curvature & & & & 1 & $\begin{array}{c}.98 \\
(.96-.99)\end{array}$ & $\begin{array}{c}.95 \\
(.93-.98)\end{array}$ \\
\hline Quadratic curvature & & & & & 1 & $\begin{array}{c}.98 \\
(.95-.99)\end{array}$ \\
\hline Cubic curvature & & & & & & 1 \\
\hline
\end{tabular}

is of theoretical interest. Here, we opted for the former method. Because the function is not restricted to the data points that were used to determine its shape, it may well be that either the maximum or the minimum lies outside the range of the observed movement. This will be the case with clearly single-curved saccades. In these cases, the first deviation is set to the curvature observed at the maximum or the minimum within the range of the movement, and the second deviation is simply set to zero. ${ }^{3}$

Maximum curvature, area curvature, and cubic curvature were all expressed in terms of percentage points of the amplitude (i.e., the number of pixels of the straight line between the start and the end of the saccade). Finally, correlation coefficients between the six measures were calculated for each individual observer. Table 1 lists the average correlations and their ranges.

\section{RESULTS AND DISCUSSION}

The $R^{2}$-distributions obtained following polynomial fitting to the saccades are shown in Figure 2. It can be seen that the linear fit does not represent the trajectories very well. The largest increase in $R^{2}$ is obtained by introducing an extra component that accounts for a single curve in the trajectory. However, it is clear that there is a population of saccades that is still not well accounted for by the quadratic function, and this population seems to have been de- creased by fitting the third-order function. This population may well be a subset of double-curved saccades (as is illustrated in Figure 1, right panel).

The simulation resulted in normal distributions of median $R^{2}$ values that indicate the goodness of fit that would be expected if fitting a higher order function simply accounted for sampling noise. The means (and standard deviations) of these distributions for the quadratic, cubic, and quartic fits were $.230( \pm .011), .796( \pm .008)$, and .912 ( \pm .004$)$, respectively. Testing the observed medians against these values showed that for the quadratic $(z=38.08, p<$ $.0001)$ and cubic $(z=4.65, p<.0001)$ functions, the improvements in goodness of fit were not due only to the accommodation of noise. The observed $R^{2}$ for the quartic fit is actually somewhat smaller than that obtained with the simulation, suggesting that the improvement from the third- to the fourth-order fit was not indicative of any systematic component in the saccade trajectories. These results support the existence of a small population of doublecurved saccades. We will return to this issue below.

Earlier, we argued that the quadratic coefficient may be directly interpreted as the deviation (in pixels or degrees) from a straight line between the predicted start and the

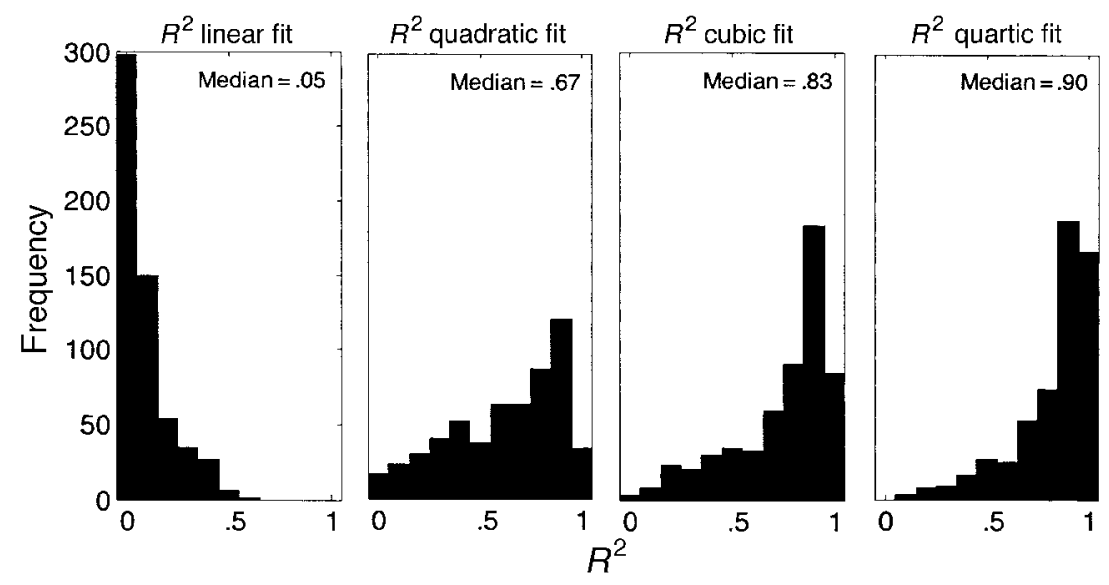

Figure 2. Distributions of $\boldsymbol{R}^{2}$ goodness-of-fit statistics for the linear fit and the second-, third-, and fourth-order polynomials. Given the strongly skewed shape of the distributions, the medians are given as a measure of central tendency. 

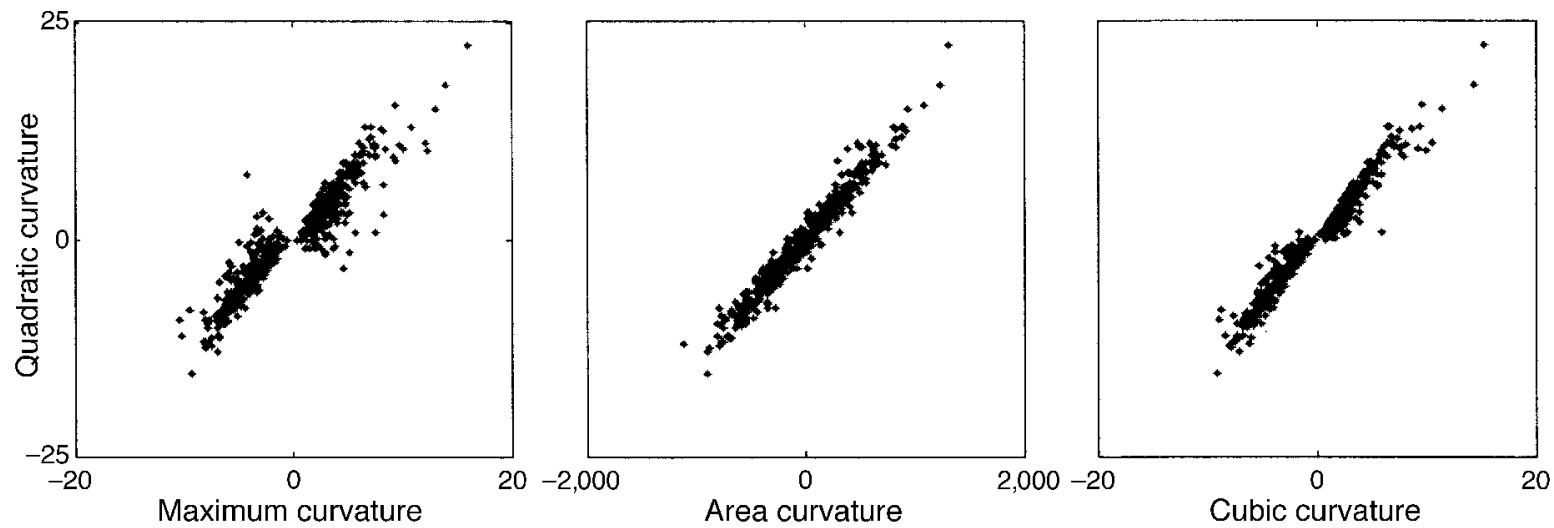

Figure 3. Associations of quadratic curvature with maximum curvature, area curvature, and cubic curvature. Note that some saccades that have been assigned close to zero curvature with the quadratic fit are curved according to the maximum and cubic curvature measures.

predicted endpoint of the saccade. This approximation holds to the extent that the mean of the predicted deviations at the start and the end of the movement are zero. On a trial-by-trial basis, departures from zero can be frequently seen. However, the mean predicted deviation ( $M=0.02$ averaged over observers) did not differ significantly from zero $[t(11)=0.62]$.

Table 1 presents a list of the average correlations (and their ranges) between the different metrics as computed for this data set. Both measures based on the initial deviation (initial direction and average) corresponded closely with each other $(M=.91)$. The associations between these two measures and the remaining four were only moderate $(M \mathrm{~s}=.70-.77)$. This pattern of correlations suggests that the initial deviation metrics measure something qualitatively different than the other metrics do, because they do not utilize all the information that is contained within the saccade trajectory.

The correlation between the area-based measure and curvature based on the quadratic fit was particularly strong $(M=.98)$. Even though the goodness of fit for the secondorder function was far from perfect, it seems to extract the same information as does the area-based measure. As was outlined earlier, a symmetrically double-curved saccade would be assigned almost zero curvature according to the area-based measure. Fitting a function with only one maximum or minimum on such a trajectory yields an almost straight line, and its quadratic coefficient will be very small. It seems that both metrics effectively calculate the dominant direction and amount of curvature in a saccade, taking the entire trajectory into account.

Figure 3 illustrates the association of quadratic curvature with the maximum, area, and cubic curvature measures. Note the clusters of points along the horizontal meridian in the left and right panels. These are the movements that have been assigned close to zero curvature with the quadratic fit, but that are curved according to the maximum and cubic metrics (sometimes to a considerable extent). Indeed, these metrics seem always to produce some curvature, creating a discontinuity at the zero point (giving the impression of two distinct clusters). The discontinuity is notably absent in the scatterplot of area against quadratic curvature.

The population of saccades with quadratic curvature close to zero consists of trajectories that are genuinely straight and those that contain a double curve. The thirdorder fit allowed for a more direct assessment of the occurrence of the latter type of trajectories. Although the

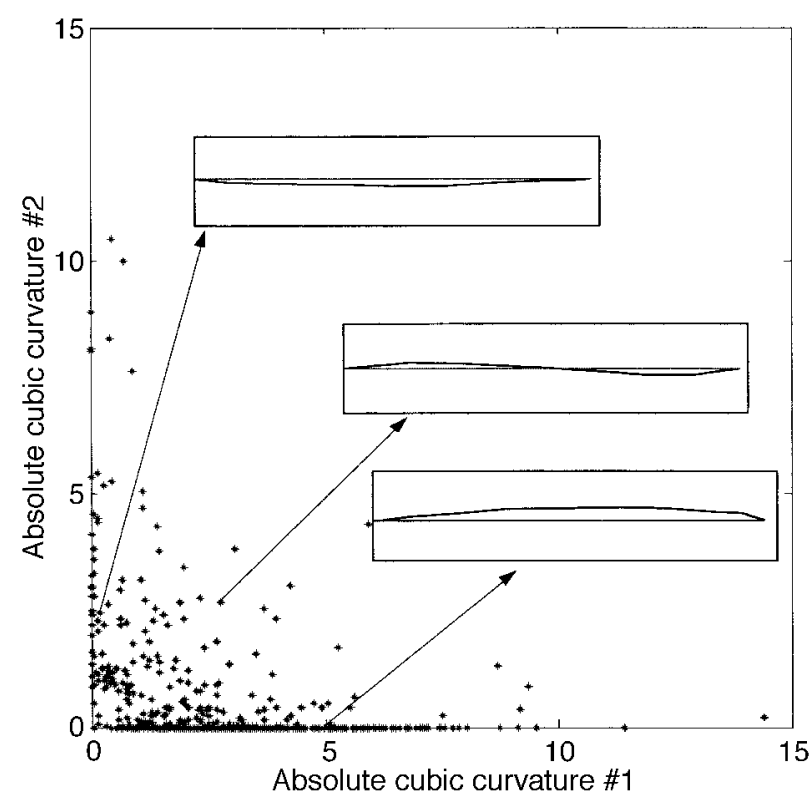

Figure 4. Cubic curvature. The absolute deviation at the first maximum or minimum in the trajectory is plotted against the absolute deviation observed at the second (if the second deviation is present within the range of the movement). The numbers on the axes are percentage points of the saccade amplitude (generally around $6^{\circ}$ ). The individual saccade trajectories corresponding to three data points are illustrated in the insets. 
cubic function has both a maximum and a minimum, for a single-curved saccade one of these (the second in our analysis) lies outside the range of the observed movement, and its associated deviation was set to zero. If the movement has a significant double curve, both the maximum and the minimum of the function will fall within the range of observed trajectory, and two deviations are computed. Figure 4 depicts the absolute deviation derived at the first maximum or minimum (cubic curvature \#1) against that derived at the second (cubic curvature \#2). It is clear that the majority of the saccade trajectories contain a single curve (points along the horizontal and vertical zero-axes). The points along the vertical zero-axis have a negligible first curve, and then a stronger curve in the opposite direction. The cluster of points with obvious positive values on both axes represents a population of saccades that are curved in both directions.

One potential problem for the curve-fitting methodology (and for the area curvature metric, for that matter) consists of saccade trajectories that show a small-often just a fraction of a pixel in the cases we have examinedreversal or a pause along the straight-line axis (i.e., the abscissa in our analysis and figures). For instance, we have noticed the occurrence of samples within a saccade in which $x_{\mathrm{i}}<x_{\mathrm{i}-1}$ (i.e., reversal along the straight line) or $x_{\mathrm{i}}=x_{\mathrm{i}-1}$ (i.e., two samples with the same $x$-coordinates but different $y$-coordinates). These phenomena are particularly prevalent at the end of the movement (when velocity drops off sharply), but they also occur midway through the trajectory. The problem is that the curve fitting is based on the spatial, but not the temporal, order of the samples. Thus, for a trajectory with a small reversal along the $x$ axis, the algorithm would try to fit a function on the samples $\left(x_{\mathrm{i}}, y_{\mathrm{i}}\right)$ and $\left(x_{\mathrm{i}-1}, y_{\mathrm{i}-1}\right)$, in that order, which does not reflect the actual order of these data points.

One solution is to consider the two samples in question as a single data point (by averaging $x_{i}$ and $x_{i-1}$, and $y_{i}$ and $\left.y_{\mathrm{i}-1}\right)$, before fitting the curve. We have carried out this analysis for the second-order fit and compared the resultant measure of quadratic curvature with those obtained using the uncorrected trajectories. Over the 12 observers, the mean uncorrected and corrected quadratic curvature values were -0.2351 and -0.2357 , respectively $[t(11)=$ 0.39, n.s.]. In addition, the individual correlations between the two measures ranged from .997 to 1 . It is important to be aware of the potentially disruptive effect of the reversals and pauses along the straight-line path. However, on the basis of our analysis, we believe the quadratic curvature metric is robust enough to allow these phenomena to be ignored for practical purposes. 4

\section{CONCLUSIONS}

The aims of this study were to (1) systematically compare the curvature metrics used in previous research, (2) develop new measures based on curve fitting, and (3) characterize the shape of saccade trajectories in a large population of eye movements. It appears that the vast majority of eye movements are curved in only one direction, an assumption that is particularly crucial for the maximum curvature measure. However, there is a separate population of eye movements that contain a double curve. Our approach allows for an investigation into the functional significance of these trajectories. (There is evidence that initial deviations in the trajectories are sometimes overcompensated; see Quaia, Pare, Wurtz, \& Optican, 2000).

The analyses presented here demonstrate that both the area-based measure and the quadratic curvature metric are effective ways of dealing with both single- and doublecurved trajectories. These measures take into account the entire movement and determine the dominant direction and amount of curvature. Given that the quadratic measure makes no assumption about the symmetry of curves within trajectories and that it results in a meaningful and readily interpretable parameter ( pixels or degrees of deviation from the straight line between saccade start and endpoint), our preference lies with this metric.

More accurate eye tracking systems (e.g., the scleral search coil) may provide more accurate estimates of maximum curvature and other measures that have been reviewed here. The curve-fitting method can easily be applied to data generated by systems other than the one we have used (with the exception of dual Purkinje image eye trackers that are less suitable for estimating eye position during the saccade; see Deubel \& Bridgeman, 1995). Regardless of the equipment used, we believe it is desirable to measure curvature taking the entire movement trajectory into account. Finally, we would like to emphasize that this approach can be extended to other types of movement that can be represented in two dimensions, such as handpointing movements.

\section{REFERENCES}

Darlington, R. B. (1990). Regression and linear models. New York: McGraw-Hill.

Deubel, H., \& Bridgeman, B. (1995). 4th Purkinje image signals reveal eye lens deviations and retinal image distortions during saccades. Vision Research, 35, 529-538.

Doy Le, M. C., \& WALKeR, R. (2001). Curved saccade trajectories: Voluntary and reflexive saccades curve away from irrelevant distractors. Experimental Brain Research, 139, 333-344.

DoY LE, M. C., \& WALKER, R. (2002). Multisensory interactions in saccade target selection: Curved saccade trajectories. Experimental Brain Research, 142, 116-130.

Findlay, J. M., \& HarRis, L. R. (1984). Small saccades to double-stepped targets moving in two dimensions. In A. G. Gale \& F. Johnson (Eds.), Theoretical and applied aspects of eye movement research ( $\mathrm{pp} .71$ 78). Amsterdam: Elsevier.

Ludwig, C. J. H., \& Gilchrist, I. D. (2002). Stimulus-driven and goaldriven control over visual selection. Journal of Experimental Psychology: Human Perception \& Performance, 28, 902-912.

McPeeK, R. M., \& Keller, E. L. (2001). Short-term priming, concurrent processing, and saccade curvature during a target selection task in the monkey. Vision Research, 41, 785-800.

Quaia, C., Pare, M., Wurtz, R. H., \& Optican, L. M. (2000). Extent of compensation for variations in monkey saccadic eye movements. Experimental Brain Research, 132, 39-51.

Sheliga, B. M., Riggio, L., Craighero, L., \& Rizzolatti, G. (1995). Spatial attention-determined modifications in saccade trajectories. NeuroReport, 6, 585-588. 
Sheliga, B. M., Riggio, L., \& Rizzolatti, G. (1994). Orienting of attention and eye movements. Experimental Brain Research, 98, 507522.

Sheliga, B. M., Riggio, L., \& Rizzolatti, G. (1995). Spatial attention and eye movements. Experimental Brain Research, 105, 261-275.

Smit, A. C., \& VAN Gisbergen, J. A. M. (1990). An analysis of curvature in fast and slow human saccades. Experimental Brain Research, 81, 335-345.

Tipper, S. P., Howard, L. A. \& Paul, M. A. (2001). Reaching affects saccade trajectories. Experimental Brain Research, 136, 241-249.

Van Gisbergen, J. A. M., Van Opstal, A. J., \& Roebroek, J. G. H. (1987). Stimulus-induced midflight modification of saccade trajectories. In J. K. O'Regan \& A. Levy-Schoen (Eds.), Eye movements: From physiology to cognition (pp. 27-36). Amsterdam: Elsevier, North-Holland.

Viviani, P., Berthoz, A., \& Tracey, D. (1977). The curvature of oblique saccades. Vision Research, 17, 661-664.

YARBuS, A. L. (1967). Eye movements and vision. New York: Plenum.

\section{NOTES}

1. In Van Gisbergen et al. (1987), observers made $40^{\circ}$ saccades, whereas $8^{\circ}$ saccades were required in the studies by Sheliga, Riggio, Craighero, and Rizzolatti (1995) and Sheliga et al. (1994, 1995). The duration of the saccade varies with saccade amplitude. As a result of this relation, the intervals at which the initial direction was sampled differed in these studies (20 msec in Van Gisbergen et al., $10 \mathrm{msec}$ in Sheliga's experiments). Our data set consisted of saccades to $6^{\circ}$ targets, and we have based our calculations of these metrics on the first $8 \mathrm{msec}$ of the movement.

2 . The MATLAB functions that were written to carry out the curve fitting, Monte Carlo simulation, and the calculation of the different metrics are available from http://eis.bris.ac.uk/ pscjhl/downloads.html. An example data set can also be downloaded for illustration of the format of the input of the programs.

3. A similar approach could be taken to calculate curvature based on the second-order fit. Here, one would compute the maximum or minimum of the function and determine its perpendicular deviation with respect to the straight line between predicted start and landing positions. Indeed, using such a measure, we found that the results are very similar to those obtained with the quadratic coefficient. The quadratic coefficient is intuitive and computationally more tractable. In addition, the quadratic coefficient is a more continuous measure, because it does not involve having to actively set the curvature of some saccades (for in stance, double-curved or genuinely straight trajectories) to zero. The latter procedure is obviously problematic when the number of these saccades differs between experimental conditions.

4. We thank Robin Walker and his colleagues for bringing this issue to our attention.

(Manuscript received February 19, 2002; revision accepted for publication October 8, 2002.) 